\title{
Arztbilder der Zukunft
}

\author{
Wie verändern sich Arztbilder in der Zukunft? Welche Erwartungen haben Chef- \\ und Klinikdirektoren, welche die Politik und die Versicherer an die Ärzteschaft der \\ Zukunft? Welche gesellschaftlichen, rechtlichen und ökonomischen Faktoren sind \\ dabei zu beachten?
}

\section{Willy Oggier}

Bilder: Peter Brandenberger

Korrespondenz:

Dr. oec. HSG Willy Oggier Gesundheitsökonomische Beratungen AG

Weinhaldenstrasse 22 CH-8700 Küsnacht

gesundheitsoekonom. willyoggier[at]bluewin.ch
Diesen Fragen widmete sich Ende September ein zweitägiges Symposium in Zürich, das gemäss Veranstalterin Doris Brandenberger-Krauer den Teilnehmern «Brain Food» geben wollte. Dies ist zweifellos gelungen: Die Veranstaltung brachte Opinion Leaders der Schweizer Ärzteschaft aus verschiedenen Bereichen zusammen.

\section{Ein Wachstumsmarkt}

Als Programmverantwortlicher des Symposiums führte der Autor dieses Artikels in seinem Eröffnungsreferat aus, dass das Gesundheitswesen ein Wachstumsmarkt bleiben werde. Allerdings heisse dies nicht automatisch, dass die etablierten Anbieter davon profitierten. Denn ein Teil des Wachstums sei gerade auch darauf zurückzuführen, dass es aus dem Misstrauen gegenüber den etablierten Anbietern generiert werde. Gleichzeitig werde die Alterung im Gesundheitswesen doppelt zu spüren sein, weil sie die Produktivität des Systems auf zwei Seiten in Frage stelle. Einerseits brauche es überproportional mehr zusätzliches Personal im Vergleich zu anderen Branchen, weil das Gesundheitswesen in einer älter werdenden Gesellschaft und angesichts der medizinisch-technischen Entwicklung einen höheren Stellenwert be- komme. Anderseits würden auch die im Gesundheitswesen Beschäftigten älter, deren körperliche Leistungsfähigkeit nehme beispielsweise in der Pflege ab. Es gehe daher vermehrt darum zu überlegen, wer die entscheidenden Kunden seien, welche Krankheiten man besetzen (und behandeln) wolle und wie man möglichst schneller als die Mit-Bewerber zu den dafür notwendigen Informationen komme.

Leitende Ärzte - Belegärzte: ein Rollenwandel Daniel Staffelbach, Rechtsanwalt bei Walder Wyss \& Partner in Zürich und selbst als Verwaltungsrat in verschiedenen Spitälern tätig, stellte die juristischen Eigenheiten des Leitenden Arztes und des Belegarztes dar. Er wagte die Prognose, dass die künftige SollBruchstelle nicht zwischen Chef- / Leitendem Arzt und dem Belegarzt, sondern zwischen im Spital tätigen und ambulant tätigen Ärzten zu stehen kommen werde. Work-Life-Balance werde dazu führen, dass sich die Arbeitszeit des Belegarztes derjenigen des Leitenden Arztes annähere. Qualitätskontrollen, Spitalplanung, die politischen Bestrebungen im Bereich der hochspezialisierten Medizin würden zu einer Zentralisierung der interessanten Operationen an weniger Spitälern führen. Und die Sub-Spezialisie-

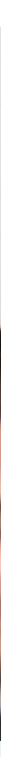

Interessant und kurzweilig fand das Publikum die Vorträge zu den vielfältigen Rollenänderungen von Ärzten und Patienten. 


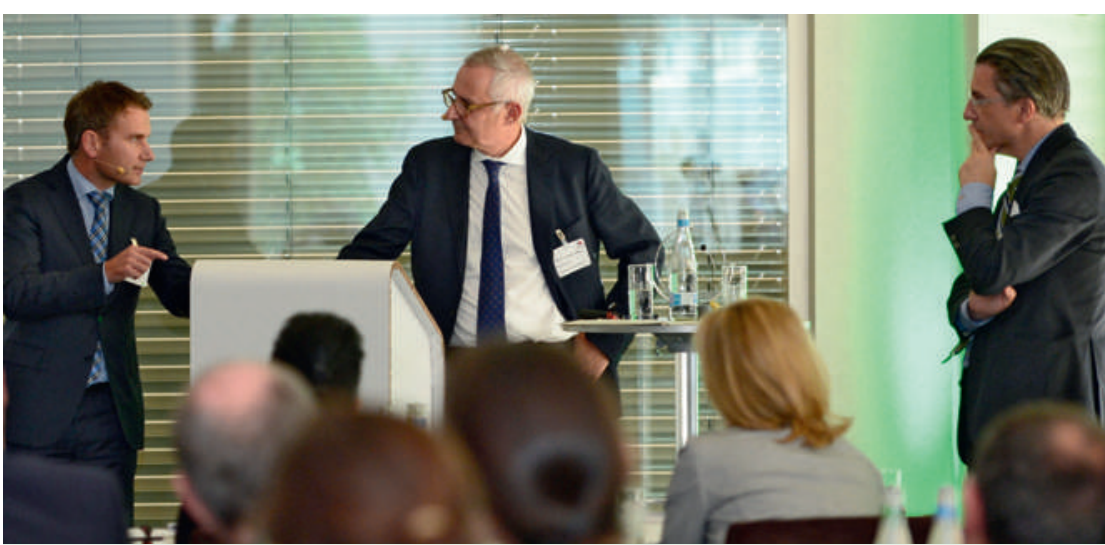

Daniel Liedtke, Andreas Tobler und Willy Oggier (v. I.n. r.) bei einer der zahlreichen Diskussionen unter den Referenten.

\section{Neue Strukturen für Universitätsspitäler}

Prof. Dr. Andreas Tobler, Ärztlicher Direktor des universitären Inselspitals in Bern, machte in seinen Ausführungen darauf aufmerksam, dass die Medizin der Zukunft die Strukturen der Universitätsspitäler massiv in Frage stelle. Gefordert sei vermehrt ein Wegkommen von hierarchischem Denken hin zu partizipativen Strukturen. Im Hybrid-OP könne man dies im Fall von Herzchirurg und Kardiologe schon bildlich sehen. Noch deutlicher sei dies in der Genomspezifizierung, wo die Sequenzierungskosten rapide sinken. Damit werde bereits die organzentrierte Struktur wieder in Frage gestellt. Es gebe heute Medikamente, die ähnliche Wirkmechanismen beim Lungenkarzinom und beim Brustkrebs ausfindig machen konnten. Spitäler müssten sich daher vermehrt für systemische Strukturen interessieren und auf fluidere Systeme einstellen. Kommunikation werde wichtiger. Medizinische Lebenszyklen würden kürzer. Es brauche aber bei aller Kritik auch die Ökonomisierung, weil die Ressourcen knapp werden («sonst bräuchte es die Ökonomisierung ja gar nicht»). Qualitativ und ökonomisch erfolgreiche Spitäler mit hoher Patientenzufriedenheit würden im Übrigen mit mehr Ärzten als Manager in der Führung als Ökonomen einhergehen.

\section{Gefragt: Spitalmanager}

In der anschliessenden Podiumsdiskussion bemerkte Dr. Marc Kohler, Direktor der Spital Thurgau AG, dass man heute fast keine Ärzte mehr habe und diese nicht alles machen könnten. Daher brauche es Spitalmanager. Dr. Urs Stoffel, FMH-Zentralvorstandsmitglied, merkte selbstkritisch an, dass die Spezies Arzt eine ganz spezielle Gattung sei und oft meine, mit dem Abschluss des Medizinstudiums hätten sie gerade auch Recht und Ökonomie studiert. Diese Zeiten seien vorbei. Dr. Matthias Winistörfer, früher selbst Arzt, heute Direktor des Zuger Kantonsspitals, plädierte für Realitätssinn. Natürlich würde er sich wünschen, dass alle Ärzte im Spital auch Manager seien, aber heute bliebe nach einer ersten Sichtung der eingehenden Bewerbungen für Chefarzt-Posten oft nur noch gar keiner oder ein Kandidat übrig, der die wichtigsten Kriterien erfülle. Sein Spital habe daher begonnen, Führungskräfte nicht nur aus der Ärzteschaft, sondern auch aus anderen Berufszweigen in betriebswirtschaftlichen Fragen zu schulen.

\section{Rollenänderungen bei Arzt und Patient}

Am zweiten Kongresstag stellte Dr. Jürg Schlup, Präsident der FMH, seine Sicht der Dinge dar. Die Möglichkeiten, Teilzeit zu arbeiten, hätten sich in den letzten Jahren angesichts der Feminisierung des Arztberufs auch in den Spitälern verbessert. Im ambulanten Bereich sei dies schon früher geschehen. Die Rolle des Arztes wandle sich vom «Halbgott in Weiss zum Behandlungspartner». Die Veränderung des Morbiditätsspektrums habe einen Einfluss, weil 
die Rolle des Patienten wichtiger werde, wenn chronische Krankheiten zunähmen. Ein passiver Patient könne nicht erwünscht sein, denn dieser interessiere sich auch nicht für Prävention. Ein weiterer Trend sei, dass Ärzte zu Beurteilten werden. Internet-Bewertungen seien im Kommen und würden sowohl von Männern wie Frauen vorgenommen, vor allem aber von Jüngeren. Es sei davon auszugehen, dass künftig auch vermehrt ältere Menschen dies tun werden. Folge davon sei leider, dass Ärzte zu Beklagten würden. Vehement verteidigte der oberste Schweizer Arzt die Rolle des Arztes als Helfer und Behandler: «Ärzte sind dazu da, Menschen zu helfen und nicht zu töten.» Er erteilte damit auch dem Arzt als Suizidhelfer eine klare Absage. Es sei eine gesellschaftspolitische und keine ärztliche Diskussion, ob man mit solchen Vorschlägen gesunde Alte unter Druck setzen wolle. Die Weiterentwicklung von Interdisziplinarität und Interprofessionalität verlange vom Arzt vermehrte Teamorientierung. Dies dürfe aber nicht so missverstanden werden, dass andere Gruppierungen ärztliche Aufgaben übernähmen, für die sie nicht ausgebildet seien und für die sich dann möglicherweise noch zusätzlich Haftpflichtprobleme für die Ärzte ergäben. Der Arzt habe heute die Koordinationsfunktion in der Behandlung. Dies sei der Grundgedanke des Krankenversicherungsgesetzes und dies solle auch so bleiben.

\section{Auswirkungen des gesellschaftlichen Wandels}

Dr. Carlo Conti, ehemaliger Regierungsrat und Gesundheitsdirektor des Kantons Basel-Stadt, heute auf Gesundheitsrecht spezialisierter Anwalt bei Wenger Plattner in Basel, betonte die gesellschaftlichen Veränderungen in der Schweiz und die damit verbundenen Herausforderungen für die Ärzte-Rolle. Dazu gehörten die Zunahme von Single-Haushalten, hohe Scheidungsraten, die erhöhte berufliche Mobilität mit der Konsequenz der Solidarität auf Distanz. Was die Ausbildung betreffe, hätten die Universitäts-Rektoren die Medizin nicht so gern, weil sie dort angesichts der auch von Liedtke erwähnten Doppelrollen nicht selbstständig entscheiden könnten. Angesichts des medizintechnischen Fortschritts verändere sich das Patientenbild, die Erwartungshaltung steige. Chronisch und mehrfach kranke Menschen seien auf wohnortnahe integrierte und ambulante Versorgung angewiesen. Die Solidarität komme zudem auf den Prüfstand. Es werde auch in Zukunft zu wenig Ärzte geben. Die Förderung von neuen Versorgungsmodellen sei daher unerlässlich. Damit stelle sich auch die Frage nach dem Skill Mix. Altersmedizin werde zum Wachstumsbereich. Dort brauche es mehr Geld und mehr Infrastruktur. Auch die ärztliche Ausbildung müsse sich danach ausrichten. Einzelpraxen

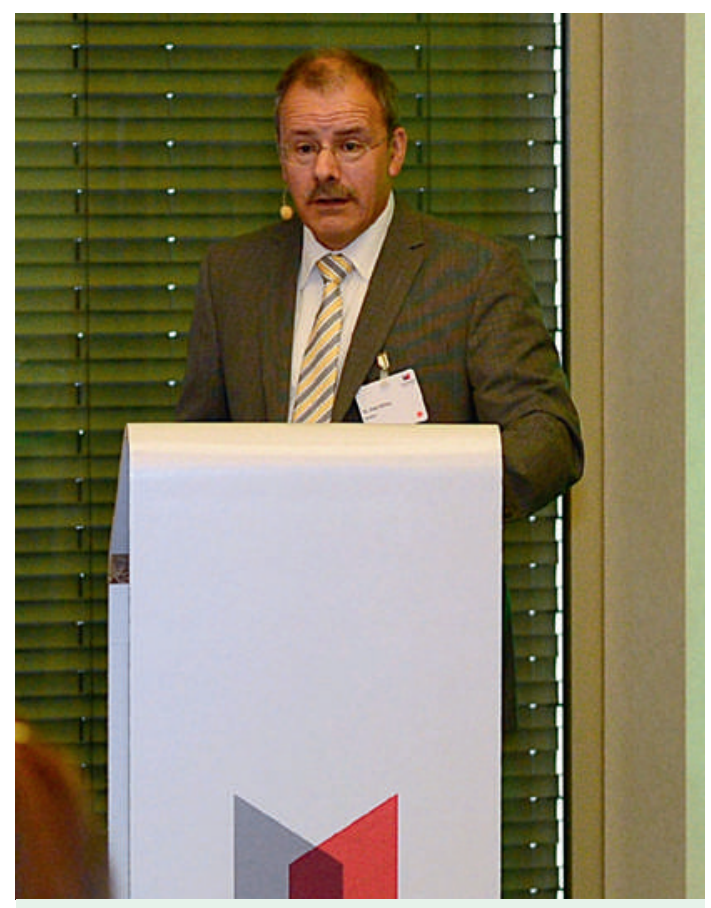

Vom «Halbgott in Weiss zum Behandlungspartner», so beschrieb FMH-Präsident Jürg Schlup eine der vielen Rollenveränderungen von Ärztinnen und Ärzten.

seien passé, Spitalambulatorien würden zu Koordinatoren der integrierten Versorgung. Die technischen Fertigkeiten der Ärzte würden vorausgesetzt, soziale Kompetenz komme dagegen als neues Selektionskriterium dazu. Gesundheits- und Sozialpolitik vermischten sich zusehends. Das Gesundheitswesen müsse sich dagegen wehren, dass ihm die Sozialkosten angehängt würden, denn dies würde nur den Kostendruck auf die Akteure im Gesundheitswesen erhöhen.

In der Schlussrunde waren sich der Vize-Präsident der FMH, Dr. Ernst Gähler, der Generalsekretär der fmCh, Dr. Markus Trutmann, und der Leiter Leistungsmanagement der Helsana Versicherungen, Dr. Michael Willer, einig, dass der Stellenwert der Zusatzversicherungen auch im ambulanten Bereich erhöht werden sollte. Die Tarifstrukturen der obligatorischen Krankenversicherung drohten, sowohl im ambulanten als auch im stationären Bereich, immer mehr verpolitisiert zu werden. Ärzte hätten daher in diesem Feld ein enges regulatorisches Konzept, wie selten eine Berufsgruppe.

Fazit der Veranstaltung: Es gibt verschiedene Möglichkeiten, das eigene Rollenbild als Arzt in der Zukunft zu gestalten. Aber wie einleitend gesagt wurde: «Man kann sich nicht nicht entscheiden. Sonst entscheidet die Politik.» 